\title{
Schweizerische Gesellschaft der Vertrauensärzte SGV
}

\section{Jürg Zollikofer}

Präsident SGV

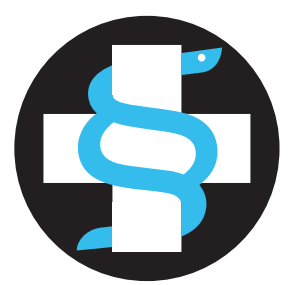

Korrespondenz:

Dr. med. Jürg Zollikofer

Im Zinggen 1

CH-8475 Ossingen

juerg.zollikofer@hin.ch

Die Schweizerische Gesellschaft der Vertrauensärzte (SGV) ist die Fachgesellschaft der FMH der Versicherungsmediziner. Mitglieder sind ausschliesslich Ärztinnen und Ärzte, die an versicherungsmedizinischen Fachfragen interessiert sind. Ursprünglich ein Zusammenschluss der für die Krankenversicherer tätigen Vertrauensärzte, hat sich die SGV in den letzten

\section{SGV SSMC}

Schweizerische Gesellschaft der Vertrauensärzte

\section{Société Suisse}

des Médecins-Conseils

Società Svizzera dei Medici Fiduciari
Jahren zu einer das gesamte Gebiet der Versicherungsmedizin umfassenden Vereinigung entwickelt. Sie befasst sich mit allen gesundheitspolitischen Fachfragen, die versicherungsmedizinische Aspekte betreffen, insbesondere der Heilungskosten und der Risikobeurteilung. Im Auftrag von FMH und santésuisse organisiert die SGV die Weiterbildungskurse für den Fähigkeitsausweis Vertrauensarzt, in deutscher Sprache in Winterthur, in französischer Sprache an der Universität Freiburg. Etwas über 260 Fähigkeitsausweise wurden bisher erteilt.

Gemäss ihrem Leitbild ist die SGV treibende Kraft in der Schaffung von Empfehlungsgrundlagen im versicherungsmedizinischen Bereich. Sie ist mit ihrer Fachkompetenz mitbestimmend in Fragen der Versicherungsmedizin. Ihre Stellung im Gesundheitswesen entspricht dem Status einer medizinischen Fachgesellschaft. Als solche präsentiert sie sich aktiv der FMH, den Partnern und der Öffentlichkeit. Im Bereich versicherungsmedizinischer Fachfragen übernimmt sie eine führende Rolle. Die Versicherungsmedizin ist die Synthese von fachmedizinischen Fakten mit den Regeln der Gesetze und Verordnungen unter Berücksichtigung des medizinischen Fortschrittes, ökonomischer Begebenheiten und ethischer Gesichtspunkte.

Die Fachkommission erarbeitet evidenzbasierte Grundlagen in versicherungsmedizinischen Fragen. Die Kommission für Weiter- und
Fortbildung ist zuständig für die kontinuierliche, kompetente und wissenschaftlich anerkannte Weiter- und Fortbildung.

Die SGV betreibt eine Homepage (www. vertrauensaerzte.ch bzw. www.medecinsconseils.ch), wo unter anderem Empfehlungen ihrer Fachkommission oder sämtliche Gesetze und Verordnungen leicht gefunden werden können. In ihrem geschlossenen Teil betreibt sie unter anderem ein allen Mitgliedern offenstehendes Forum.

Die Mitglieder der SGV, also Vertrauensärzte der Krankenversicherer, Ärzte der Suva, der RAD, der MEDAS oder beratende der Privatversicherer wie auch von Firmen und Behörden, verstehen sich in erster Linie als Ärzte und dadurch als Mittler zwischen Versicherer, Leistungserbringer und Patient. Dabei sind sie es durchaus auch gewohnt, manchmal eben nicht als Mittler, sondern als Interessenvertreter der anderen Seite wahrgenommen zu werden: für Leistungserbringer der Versicherer, für die Versicherer der Leistungserbringer.

Die SGV ist Herausgeberin des «Manuals der Vertrauensärzte». Momentan laufen die Arbeiten für die 3. Auflage. Das «Manual» kann als Handbuch der Sozialversicherungen bezeichnet werden.

Die SGV führt als Hauptanlass den Jahreskongress durch, der am 16. April 2008 in Freiburg Workshops zu verschiedensten Themen anbietet. Der zweite Tag ist immer einem bestimmten Thema gewidmet. Am 17. April wird intensiv über Fragen des Datenschutzes diskutiert werden. Sowohl der Eidgenössische Datenschutzund Öffentlichkeitsbeauftragte Hanspeter Thür, wie auch der Ombudsmann der Krankenversicherer Rudolf Luginbühl und FMH-Präsident Jacques de Haller, werden neben Vertretern der Patientenorganisationen und der Versicherer referieren. Die Veranstaltung ist öffentlich. Genaueres siehe: www.vertrauensaerzte.ch. 